\title{
Algunas notas sobre los actores de las relaciones internacionales en la actualidad
}

José Manuel Canales-Aliende'

Recibido: 21/05/2014 - Aceptado: 30/05/2014

\section{Resumen}

El presente artículo tiene como objetivo mostrar la crisis múltiple del Estado, el cambio del modelo y de la praxis de las relaciones internacionales y sus actores, poniendo de relieve una serie de hechos relevantes, que son sin duda retos que se deben afrontar por todos los sistemas político-administrativos; y aquellos son: a) la revitalización del conflicto político, por razones económicas; b) la práctica de un pensamiento individualista y el darwinismo social, dejando atrás los principios de igualdad y equidad social, provocando la fractura social; c) lo que enfatiza la debilidad de la democracia como régimen político legítimo para dar respuestas a la sociedad; d) a lo que debemos añadir un proceso de mutación de valores; d) junto a la aparición de una actitud de "conservadurismo del miedo"; e) y el fin de la separación clásica entre "política interior" y "política exterior".

\section{Palabras clave}

Relaciones internacionales, gobernanza, sistemas político-administrativos, crisis económica.

\begin{abstract}
This article aims to show the multiple crises of the State, and the change in the model and praxis of international relations and its actors, by highlighting a number of significant facts, which certainly represent a challenge for all political- administrative systems. These include: a) the revitalization of the political conflict due to economic reasons, b) the practice of an individualistic thinking and social Darwinism, leaving behind the principles of equality and social equity thus causing social fracture, c) the factors which emphasize the weakness of democracy as a legitimate political regime to respond to society d) a mutating process of values; d) the appearance of an attitude of "fear conservatism," e) and the end of the classical separation between "internal politics" and "foreign policy".
\end{abstract}

\section{Keywords}

International relations, governance, politicaladministrative systems, financial crisis.

1 José Manuel Canales Aliende. Doctor en Derecho. Catedrático de Ciencia Política y de la Administración de la Universidad de Alicante y director del Grupo de Investigación Observatorio Lucentino de Administración y Políticas Públicas Comparadas. Consultor de la Ocde, del Banco Mundial y de la Unión Europea. Profesor visitante de numerosas universidades, de EE.UU. (Universidad de Georgetown), de Europa (Bath, Instituto de Estudios Políticos de Burdeos IV) y de Latinoamérica (entre otras, la Universidad de Santa María La Antigua, la Universidad Nacional de Panamá, la Universidad Nacional de Colombia, la Universidad Nacional de Costa Rica y la Universidad Nacional del Cuyo en Mendoza, etc.). Autor de más de un centenar de obras y artículos académicos, entre los que destacan: Panorama actual de la Ciencia de la Administración (Inap, 1987); Lecciones de Administración y de Gestión Pública (Universidad de Alicante, 2002); Documentos básicos para la Modernización y el Fortalecimiento de las Administraciones en Iberoamérica (ECU, 2009); 0, Canales Aliende, J. M. \& Menéndez Alzamora, M. Sistema político y administrativo valenciano (Tirant lo Blanch, 2002). Funcionario excedente de varios cuerpos superiores de diversas administraciones públicas españolas. im.canales@ua.es 


\section{I) Introducción y planteamiento general}

En la sociedad actual, caracterizada, entre otras, por las notas de: complejidad, diversidad, dinamicidad, inseguridad y postindustrialismo $-\mathrm{y}$ ello en un contexto nuevo estructural y problemático de la globalización y de la economía del conocimiento-, se han producido numerosas transformaciones esenciales $y$ relevantes del sistema político-administrativo heredado del siglo $\mathrm{XX}$ pasado. Algún autor, como Beck, habla de "la segunda modernidad", como etapa superadora y transformadora de la previa, de la "postmodernidad".

Dentro de las mutaciones acontecidas en el seno de los sistemas político-administrativos, habría que destacar sin duda la crisis múltiple del Estado, y el cambio del modelo y de la praxis de las relaciones internacionales, fruto de lo anterior.

En el modelo precedente, el sistema político y las relaciones internacionales se basaban en la centralidad y la preeminencia del papel del Estado; y a su vez las relaciones internacionales se basaban esencialmente en tres principios: 1) el de soberanía nacional, fruto de la Paz de Westfalia de 1648; 2) el de territorialidad, que implicaba la delimitación clara y segura de las fronteras nacionales; y 3 ) el de nacionalidad, en virtud del cual se concedía el derecho de ciudadanía a cada persona nacida en cada país.

El Estado contemporáneo, configurado en base a los tres principios fundamentales antedichos, llevaba a cabo una acción exterior o diplomática centralizada, estatalista, y monopolizada al máximo; y centrada básicamente en temas políticos y/o militares. El brazo ejecutor, o burocracia, más o menos profesional, según los países, lo constituiría el servicio exterior; siendo el Congreso y el Tratado de Viena la norma internacional básica imperante.

\section{II) El nuevo contexto mundial y sus efectos}

El mundo hoy está inmerso en una globalización o mundialización, que tiene, como es sabido, múltiples aspectos o facetas, y no solo la económica, produciendo un cambio estructural de la sociedad con las notas antedichas. Por otro lado, la globalización ha traído consigo nuevos y relevantes problemas: sociales, ecológicos, de descontrol de los medios e instituciones financieras, inseguridad, globalización, etc.
También, fruto de la nueva realidad, se ha producido una transformación y una crisis profunda del modelo del Estado y de su papel en el sistema político-administrativo, el cual deja de ser estatocéntrico y pasa a ser policéntrico.

La crisis del Estado es múltiple (a saber: fiscal, de bienestar, territorial, y de tareas y funciones); y destacaría, en especial, la territorial; o la crisis y transformación del modelo previo del Estado-nación. Este no solo ha perdido "por arriba" competencias fruto de los procesos de integración supranacional, sino también en su interior, como consecuencia de los procesos operados de descentralización político-administrativa. Además, ha cambiado del modo siguiente: a) el Estado ya no ocupa el papel primordial de los sistemas políticos, ya que estos son "policéntricos"; b) el poder político está muy extendido a escala mundial; c) la acción exterior y las políticas de los Estados en las relaciones internacionales incluyen nuevos objetivos y contenido, más allá de la primacía de lo militar y político, para abordar temas humanitarios, del medio ambiente, movimientos migratorios, etc.; d) los Estados se ven obligados a actuar como actores estratégicos, no únicos, en la defensa de sus posiciones, en un contexto y sistema global complejo de intereses de todo tipo, y e) a pesar de todo lo anterior, del Estado sigue teniendo aún algunas competencias esenciales, como: la regulación, la sanción y la seguridad. Hay, pues, un "retorno" del Estado en múltiples manifestaciones. Por otro lado, la eficacia y las funciones del Estado no se consiguen simplemente reduciendo su tamaño.

Además, la teoría clásica de la dogmática jurídica, que señalaba la existencia de tres elementos esenciales constitutivos internos del Estado, se ha visto afectada y cuestionada. Estos tres elementos eran los siguientes: a) soberanía nacional; b) competencias exclusivas y excluyentes, y c) población fija.

Se ha producido también una expansión y complejidad de las relaciones e interconexiones sociales, a través de múltiples dimensiones de actividades de todo tipo; y ello apoyado e impulsado por las nuevas tecnologías en un contexto creciente de permeabilidad de las fronteras.

Pero la globalización, además de los retos y de los nuevos problemas que plantea, y en particular el de la inseguridad, va a implicar, sobre todo y ante todo, una relevancia creciente de la diversidad y pluralidad de culturas, ideologías, valores y religiones a escalas nacional y mundial. 
Otro problema grave añadido a los anteriores, del proceso de globalización, o uno de los aspectos de la cara oscura de la globalización, es el llamado "poder blando", que es heterogéneo, difuso y por tanto incontrolable y al servicio de lobbies económicos poderosos internacionales; que pretende, entre otras finalidades, hacer de los Estados unas meras colonias sometidas al mandato de la economía mundial descontrolada.

La crisis múltiple actual, fruto del contexto de la globalización, pone de relieve también una serie de hechos relevantes, que son sin duda retos que se deben afrontar; y entre estos citaría los siguientes: a) la revitalización del conflicto político, por razones económicas; b) el individualismo y el darwinismo social, y el aparcamiento en muchas ocasiones de los principios de igualdad y equidad sociales; c) la debilidad de la democracia, como régimen político legítimo y válido para dar respuestas societarias; d) la mutación de valores; d) la aparición de una actitud de "conservadurismo del miedo", y e) el fin de la separación clásica entre "política interior" y "política exterior".

La nueva realidad se ha denominado como "política interior global", y "que no se refiere a un concepto normativo de la filosofía o de la teoría política, sino a una realidad impetuosa y salvaje que avanza por encima, por debajo, y a través de las fronteras nacionales que imperan en las mentes y en las instituciones...", según Ulrich Beck (2011).

\section{III) La pluralidad de actores intervinientes en las relaciones internacionales}

Si el modelo heredado del siglo XX implicaba un modelo único y monopolista de las relaciones internacionales, hoy día aquel está superado y es inviable, pues hay una pluralidad y diversidad creciente de sus actores y protagonistas.

Entre los nuevos actores de las relaciones internacionales, estarían en primer lugar los gobiernos y las administraciones públicas subnacionales y locales, que juegan un papel importante en la cooperación y el desarrollo económico; y en segundo término, pero no por ello menos relevante, estarían las diversas y plurales instituciones representativas de la sociedad civil: ONG, movimiento sociales y ecológicos, iglesias y movimientos religiosos varios, movimientos de defensa de derechos humanos, etc.

Los Estados, actores ya no únicos, están llamados a coordinar, bajo el principio y la orientación de la supremacía, pero no de la jerarquía, todas las actuaciones internacionales de los distintos actores de sus respectivos países en el ámbito internacional, defendiendo sus intereses, a través de una estrategia común.

Por otro lado, las relaciones interestatales, a su vez, deben fundamentarse y orientarse cada vez más hacia una cooperación y coordinación mutua mayores, fruto de la creciente e imparable interdependencia existente; y ello con la idea clara de que no existe aún, fruto de la gobernanza mundial, unas nuevas instituciones (parlamentarias y judiciales, en especial) y autoridades de ese carácter, luchando contra los poderes fácticos incontrolados.

Las Naciones Unidas, tal y como están configuradas y actúan en la actualidad, son incapaces de dar respuestas satisfactorias a los desafíos existentes en el contexto mundial; y por supuesto, el grupo del "G-20" no deja de ser un lobby estrictamente económico.

Ciertamente, la solución ideal y la respuesta a los problemas planteados por la globalización estarían en la puesta en práctica de la llamada, por David Held, "democracia cosmopolita", y también en la transformación del Estado nacional precedente en un "Estado trasnacional".

\section{IV) La nueva diplomacia pública}

La nueva diplomacia, fruto de la nueva realidad internacional, se va a caracterizar sustancialmente por ser: a) policéntrica, o plural; b) heterárquica; c) proactiva, y no reactiva, y d) por actuar gestionando redes. 
Tabla 1

Comparativo de las características de los instrumentos de la vieja diplomacia versus la nueva diplomacia

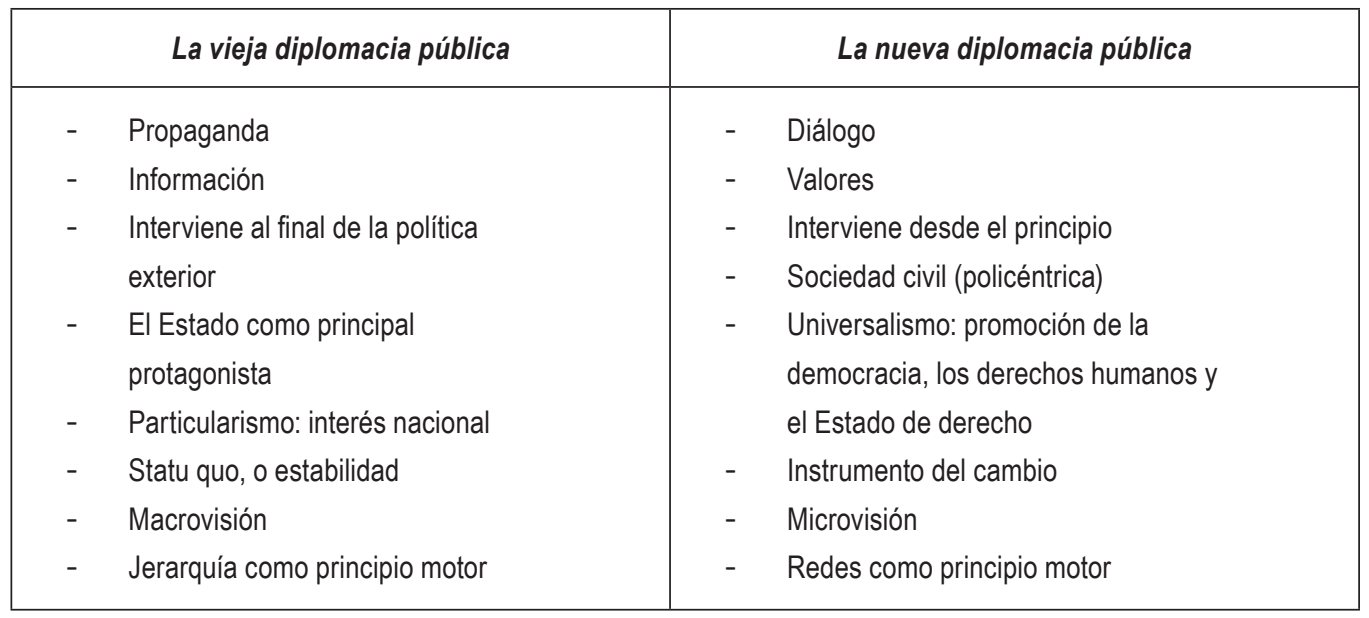

(Fuente: Elaboración propia)

En cuanto a la diplomacia estatal, representada por el Servicio Exterior, este debe modernizarse y capacitarse adecuadamente para poder afrontar los retos de la nueva era. La diplomacia clásica de "salón" corresponde a sociedades estáticas del pasado; y hoy se necesita una "diplomacia de gestión estratégica".

A su vez, el contenido de la diplomacia estatal debe ser más plural, abarcando con mayor énfasis aspectos culturales y educativos, económicos y sociales (en especial Ios problemas migratorios); de I+D, etc. La promoción de la "marca" de su país, como un valor intangible relevante, debería ser también otra de sus dedicaciones prioritarias.

Dentro del amplio y plural contenido de la diplomacia actual, destacaría la importancia creciente de la llamada "diplomacia cultural". En ese sentido, una de sus manifestaciones en la región iberoamericana se plasmaría en el documento "Elementos para una Agenda de la Diplomacia Cultural en Iberoamérica", fruto del Primer Encuentro Iberoamericano de Diplomacia Cultural, celebrado en la ciudad colombiana de Cartagena de Indias, durante el mes de marzo de 2011, a instancias de la Secretaría General Iberoamericana (Segib).

El papel y la gestión de las redes sociales, a escalas nacional e internacional, es un característico reto esencial para toda la actual diplomacia pública. Ahora bien, y sin perjuicio hoy de lo anterior, entre los objetivos prioritarios de esta nueva diplomacia pública deberían estar los siguientes: a) la democratización o calidad de la democracia; b) la consolidación de un Estado transnacional; c) la salvaguardia del medio ambiente, y c) la garantía y el fortalecimiento de los derechos humanos, en especial los de la llamada "tercera" y "cuarta" generación de estos.

\section{V) Conclusiones}

La gobernanza y la democracia cosmopolita, como procesos ideales que se deben lograr, no impiden en absoluto la existencia de un Estado que podría calificar sintéticamente del modelo siguiente: moderno pero modesto, eficaz, fuerte y con capacidad estratégica, y como expresión del pacto social solidario.

La diplomacia pública plural, debidamente capacitada, dirigida y coordinada por el Estado, será el instrumento idóneo, sin duda, para el desarrollo ordenado de las capacidades estatales en el contexto internacional actual, llevando a cabo diversas políticas públicas necesarias y adecuadas para cada momento histórico.

La capacitación y la actualización de los miembros de la diplomacia pública resulta imprescindible en la actual sociedad del conocimiento.

Por último, y sin perjuicio de lo anterior, para finalizar señalaría también que es preciso huir, en la actuación pública, tanto a escala nacional como internacional, de los planteamientos ideológicos obsoletos, fracasados y unilaterales del neoliberalismo tecnocrático y del 
neomarxismo para dar respuestas a los retos y problemas actuales. Hay que buscar nuevos valores públicos, adecuados a las demandas del presente; y hacer que el capitalismo (única organización hoy vigente de producción y actuación económica) se transforme hacia una praxis más humana y solidaria, y menos individualista y tecnocrática, en el que el tercer sector de la economía o "economía social" tenga un papel predominante.

\section{Bibliografía básica consultada}

Beck, Ulrich (1998). Qué es la globalización. Falacias del globalismo, respuestas a la globalización. Barcelona: Ediciones Paidós Ibérica, S.A.

Bech, Ulrich (2006). La sociedad del riesgo global. Madrid: Siglo XXI, Editores, S.A. 2. ${ }^{\text {a edición. }}$

Beck, Ulrich (2011). Crónicas desde el mundo de la política interior global. Barcelona: Editorial Paidós. S.A.

Bobbio, Norberto (2007). El futuro de la democracia.
México: Editorial Fondo de Cultura Económica, Cuarta reimpresión.

Held, David (1997). La democracia y el orden global. Del Estado moderno al gobierno cosmopolita. Barcelona: Editorial Paidós, S.A.

Noya, Javier (2007). Diplomacia Pública para el siglo XXI. Madrid: Real Instituto Elcano y Editorial Ariel.

Nye, J. (2003). La paradoja del poder norteamericano. Madrid: Editorial Taurus S.A.

Nye, J. (2004). Soft Power. New York: Public Affairs.

Vallespín, Fernando (2000). El futuro de la política. Madrid: Editorial Taurus.

Vallespín, Fernando (2010). "La transformación del Estado como consecuencia de los impactos de la crisis económica". En Reforma y Democracia, Caracas: Revista del CLAD, nº 48, de octubre del 2010.

Varela Barrios, Edgar (2007). La soberanía transformada. Cali: Ecoe Ediciones y Universidad del Valle. 